\title{
Optimal Output Vector Feedback Control Strategy for Wind Power Systems
}

\author{
Shahida Khatoon ${ }^{\mathrm{a}}$, Ibraheem Nasiruddin ${ }^{\mathrm{b}}$, Reshma Ehtesham ${ }^{\mathrm{a}}$ \& Ramesh C. Bansal $\mathrm{C}^{\mathrm{c}^{*}}$ \\ ${ }^{a}$ Department of Electrical Engineering, Jamia Millia Islamia, New Delhi, India \\ ${ }^{b}$ Department of Electrical Engineering, Qassim University, AI-Qassim, Saudi Arabia \\ ${ }^{c}$ Department of Electrical Electronics and Computer Engineering, Pretoria University, Pretoria, South Africa
}

\begin{abstract}
This article presents the design of optimal output feedback controllers to enhance the dynamic performance of wind turbine generator supplying an infinite bus through transmission line. Efforts have been made to propose optimal regulators based on feedback of output state variables, which are easily accessible and available for the measurement. The designed optimal output feedback controllers are implemented in the wake of step disturbance in the system. The system dynamic responses and pattern of closed loop system eigenvalues for various system states are obtained with the designed optimal regulators to investigate the system dynamic performance. The investigations of the results obtained reveal that the reduction in system states used for feedback has resulted in degradation of system dynamic performance. However, for a particular set of output variables used as feedback; five states are used for feedback for an eighth order original system. The system dynamic performance is the same as offered by optimal controllers based on full state vector feedback control strategy.
\end{abstract}

Keywords: wind power system, optimal regulator, output feedback control, closed-loop eigenvalues, dynamic system performance

\section{Introduction}

The use of electrical energy has been continuously increasing day by day everywhere. The rate of increase in energy consumption is not uniform all over the world. The trend shown by developed countries is almost technically linear, whereas it has exponential shape in most of the developing countries. To cope with increasing demands from consumers, utilities have been using all the available energy resources. In the competing energy market, they are not complying with the necessary guidelines set to protect the environment.

Among renewable sources of energy, wind has been identified as one of the most promising sources. The worldwide growth of installed wind energy capacity is at a very encouraging pace. It almost doubled every three years since 1990 and is expected to increase further due to the growing interest of the energy planners and utilities in this area. With the recent developments in technology, the use of higher capacity wind turbines has enabled utilities to develop wind farms of mega-watt capacity economically. With the increasing size of wind machines, better energy productivity is evident at lower cost. Therefore, the integration of wind power into utility systems is an area of future research interest.

Wind turbine generators possess some special characteristics that are different from other types of generating units, as reported in [1]. Many investigations on dynamic stability of wind turbine generators have been reported by various researchers [2-9]. One of the important aspects of the wind turbine generator is its effect on the stability of the system to which it is connected. Although they do not contribute to the instability of the power systems, results have indicated that there may be modal frequencies that are in the range of large interconnected power systems [6, 7]. Power system stabilizers (PSSs) can be employed to increase the damping torque of wind turbine generators. However, a PSS designed considering a particular operating condition may not yield optimal 
performance when operating conditions changes. This limitation can be overcome by using adaptive control techniques.

Undoubtedly, application of modern control theory to design optimal controllers for power systems has been the main subject of many publications over the past decades. It has been established that the dynamic performance of the system with greater stability margins can be achieved with optimal control strategies $[10,11]$. The application of modern control techniques for the design of optimal regulators depends on the availability of all the system states directly or using an observer or filter. However, in real situations, it is really difficult or sometimes impossible to measure all the system states. To circumvent these, the design of optimal regulators using easily available output states as feedback, which are easily accessible and available for measurement, is preferred. Researchers have issued a number of articles dealing with the design of optimal system controllers/regulators using the output vector feedback control concept [12-14]. However, attention is paid to propose the suboptimal controllers using feedback of output variables, and therefore, the controller designs do not offer the optimal and desired system dynamic performance.

In addition to these, a number of articles have appeared in the literature dealing with various control aspects of wind energy generators [7, 8, 15-22]. In [15], an optimal control scheme was proposed that optimizes the use of a renewable energy system to the maximum extent while limiting the use of the diesel generator. The developed control scheme reduces the operational hours of the diesel generator, thereby reducing the running cost of the hybrid energy system and pollutant emissions. In another scheme, the pitch angle and the tip speed ratio of each one of the wind turbines are optimized to minimize the overall wake effect between wind turbines and, as a consequence, maximize the power produced using a genetic algorithm [16]. An optimal control algorithm employing the direct shooting method for a multi-input-multi-output (MIMO) wind turbine system, when compared to the traditional single-input-single-output (SISO) feedback control with respect to the effectiveness of wind energy capture of the turbine system, enables more wind energy capture when the turbine operates in a narrow range of rotor velocity due to the constraint incurred by the selected electrical generator and the turbine design [17]. A state observer is designed using the barrier Lyapunov function (BLF) back-stepping procedure in conjunction with an adaptive output feedback control for a class of non-linear SISO systems to estimate the unmeasured states. Therefore, only one adjustable parameter needs to be updated, alleviating the online computation burden. The optimality of the wind power system in relation with the trade-off between the wind energy conversion maximization and the minimization of the induction generator torque variation is achieved by using a combined optimization criterion, resulting in an LQ tracking problem with an infinite horizon and a measurable exogenous variable (wind speed). The proposed optimal controller and a proposed general regression neural network (GRNN) controller are designed to drive the turbine speed to extract maximum power from the wind and adjust to the power regulation [18], maintaining system stability and reaching the desired performance even with parameter uncertainties.

A dynamic feedback controller can maximize the conversion efficiency and eliminate torque oscillations propagated through the drive train, which preserves the system structure and improve the system performance without measuring the wind velocity [19]. The design of an adaptive output feedback controller for wind turbine generators using a neural network was proposed in [21]. The results obtained with output feedback controllers were compared with those achieved with optimal controllers. However, only a set of variables has been used for the feedback in the controller design.

In view of above discussions, it has been observed that wind power plants are gaining momentum worldwide among the renewable source based power plants due to their numerous environmental, technical, and economic advantages. However, the important aspect of the wind turbine generator is its effect on the stability of the system to which it is connected. Therefore, the feasibility of wellestablished control strategies is to be tested for their implementation and investigation aspects in new environments. As discussed earlier, although optimal control designs based on full-state vector 
feedback control offer the desired system dynamic performance, practical difficulties associated with their design and physical realization have limited the scope of such schemes. On the other hand, the output feedback control technique uses only selected subsets of state variables that are conveniently and practically available for measurement and contain enough information about the complete system. As a result, when designing optimal output vector feedback control, constraints are imposed on the structure of the feedback matrix so as to affect the feedback of the desired state variables only.

This research work aims to design and investigate the feasibility of the optimal output vector feedback control strategy for wind turbine generators reported in [20,21]. The variables selected for the feedback are limited to the system states, which are easily accessible and available for measurement. These controllers also have a greater feasibility of their implementation in any practical situations. The designed optimal controllers based on the output vector feedback control technique are implemented, and the system dynamic responses for various system states are obtained and compared with that obtained with controllers designed using the full-state vector feedback control strategy. The pattern of closed-loop eigenvalues is also obtained to ensure stability of the system with the designed control strategy.

\section{Power System Model Under Investigation}

The proposed power system model is a wind turbine generator injecting the generated electrical power from wind to an infinite bus connected through a transmission link. The wind turbine generator under consideration is a 1-MVA unit, extrapolated from a 100-kW unit of the NASA-Lewis Research Centre as reported in [21] as a part of the Energy Research and Development Administration (ERDA) wind energy program. The schematic diagram of the system under investigation is given in Figure 1 . However, the detailed transfer function model of the system is shown in Figure 2.

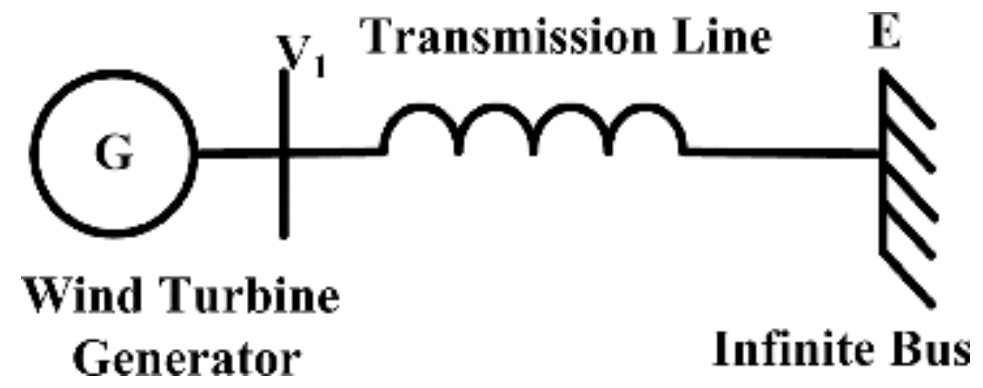

FIGURE 1. Schematic diagram of system under investigation.

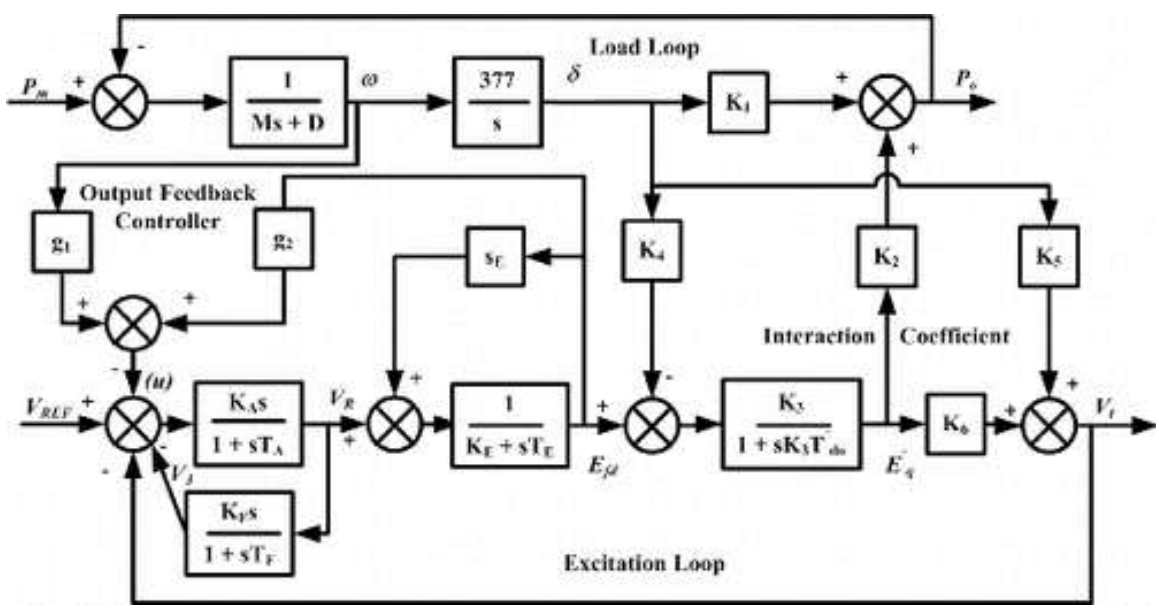

FIGURE 2. Transfer function model of wind energy generating system. 


\section{Optimal Controller Design Using Output Vector Feedback Control Technique}

The wind power system is described by a completely controllable and observable linear time-invariant state space representation as:

$$
\begin{aligned}
& \frac{d}{d t}[A x(t)]=A x(t)+B u(t)+T d(t), \\
& y(t)=C x(t) .
\end{aligned}
$$

For the output feedback control problem, obtain a control law of the form

$$
\left[U_{o u t}\right]=-\left[\psi_{\text {out }}^{*}\right] y(t),
$$

which minimizes the performance index $(\mathcal{J})$ as defined by the following relation:

$$
J=\frac{1}{2} \int_{0}^{\infty}\left[\left(x^{T}(t) Q x(t)+U_{\text {out }}^{T}(t) R U_{\text {out }}(t)\right)\right] d t,
$$

subject to the system dynamic constraints.

Equations (1), (2), and (3) yield a closed-loop system:

$$
\frac{d x}{d t}=\left(A-B \psi_{\text {out }}^{*} C\right) x,
$$

where $\left(A-B \psi^{*}{ }_{\text {out }} C\right)$ is a closed-loop system matrix.

Using Eqs. (2), (3), and (4), the structure of the performance index can be modified as

$$
J_{1}=\frac{1}{2} \int_{0}^{\infty} x^{T}\left[Q+C^{T}\left(\psi_{\text {out }}^{*}\right)^{T} R \psi_{\text {out }}^{*} C\right] x d t .
$$

Substituting the solution of Eq. (5) into Eq. (6), it is observed that the function $J_{1}$ depends upon the initial state $x(0)$.

For the completely controllable and observable system given by Eqs. (1) and (2), the function $J_{1}$ is given by

$$
J_{1}=x^{T}(0) S x(0),
$$

where $S$ is a symmetric matrix.

From Eqs. (5)-(7), the function $S$ is given by

$$
S=\int_{0}^{\infty} e^{D^{T} t} x^{T}\left[Q+C^{T}\left(\psi_{\text {out }}^{*}\right)^{T} R \psi_{\text {out }}^{*} C\right] e^{D^{T} t} d t .
$$


The matrix $S$ is obtained as a unique positive definite solution of the following Lyapunov equation [22]:

$$
D^{T} S+S D+Q+C^{T}\left(\psi_{\text {out }}^{*}\right)^{T} R \psi_{\text {out }}^{*} C=0 .
$$

To eliminate the dependence on initial state $x(0)$, an auxiliary optimization problem is formulated as follows. The function $J_{1}$ as given by Eq. (7) can be rewritten as

$$
J_{1}=\operatorname{Tr}\left[S x(0) x^{T}(0)\right] \text {. }
$$

Assuming $x(0)$ to be a random vector uniformly distributed over the surface of a unit sphere,

$$
x(0) x^{T}(0)=I \text {, }
$$

where $l$ is an $n \times n$ identity matrix. Therefore,

$$
J_{1}=\operatorname{Tr}[S] .
$$

The auxiliary optimization problem can now be stated as follows.

Obtain the feedback gain matrix $\psi_{\text {out }}^{*}$, which minimizes performance index $J_{1}$ defined by Eq. (10) subject to the constraint given by Eq. (9). By defining the Hamiltonian and equating its derivatives with respect to $P, S$, and $\psi^{*}$ out to zero, the following conditions are obtained using gradient matrices [11]:

$$
\begin{aligned}
& D^{T} S+S D+C^{T}\left(\psi_{\text {out }}^{*}\right)^{T} R \psi_{\text {out }}^{*} C+Q=0, \\
& D^{T} P+P D+I=0, \\
& \frac{\partial H}{\partial \psi_{\text {out }}^{*}}=2\left(R \psi_{\text {out }}^{*} C P C^{T}-B^{T} S P C^{T}\right) .
\end{aligned}
$$

Equations (11)-(13) are solved iteratively using a non-linear programming technique. The solution is obtained using an algorithm based on a steepest decent iterative technique. The solution of Lyapunov equations (Eqs. (11) and (12)) is obtained based on an algorithm proposed in [11]. A schematic representation of output vector feedback controller is shown in Figure 3. 


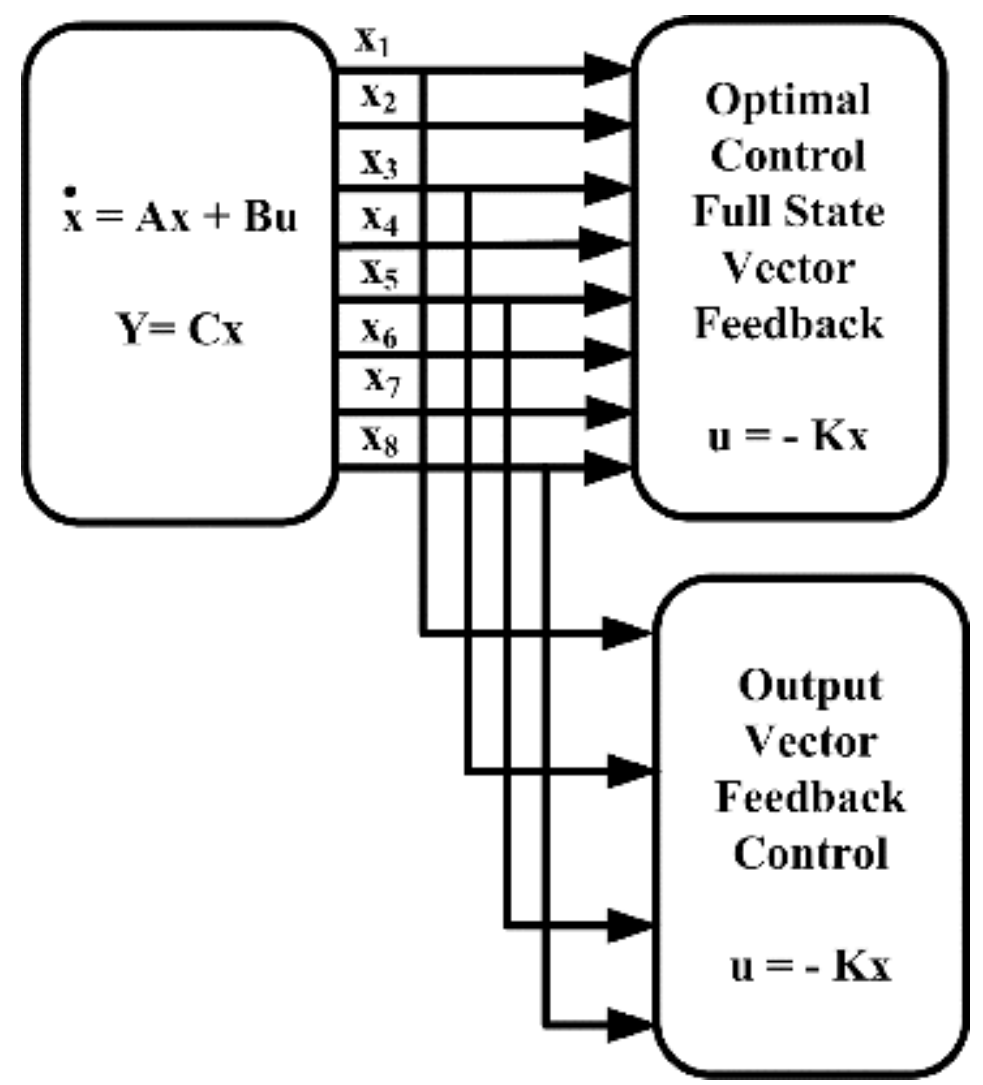

FIGURE 3. Block diagram representation of output vector feedback controller.

\section{Dynamic Model of the System}

The dynamical model of the system under consideration is given by differential equations (Eqs. (1) and (2)), respectively. The transfer function model of wind energy generating unit is developed in [21] and is shown in Figure 2. The structures of system vectors and matrices can be deduced using the following differential equations derived from the transfer function model of Figure 2:

$$
\left(P_{m}-P\right)\left(\frac{1}{s} M+D\right)=\omega \text {. }
$$

The torque angle loop in Figure 2 is a pure oscillator with zero damping due to electrical load characteristics; therefore,

$$
P_{m}-P=s M \omega,
$$

or

$$
\begin{aligned}
\frac{\left(P_{m}-P\right)}{M} & =s \omega=\omega, \\
\omega \frac{377}{s} & =\delta, \\
\text { or } \delta & =377 \omega,
\end{aligned}
$$




$$
\begin{gathered}
E_{q}^{\prime}=\left(-\delta K_{4}+E_{f d}\right)\left(\frac{K_{3}}{1+E_{f d} K_{3} T_{d o}^{\prime}}\right), \\
E_{q}^{\prime}=-\frac{K_{4} \delta}{T_{d o}^{\prime}}-\frac{E_{q}^{\prime}}{K_{3} T_{d o}^{\prime}}+\frac{E_{f o}^{\prime}}{T_{d o}^{\prime}}, \\
E_{f d}=\left(V_{R}-E_{f d} S_{E}\right)\left(1 / K_{E}+s T_{E}\right), \\
E_{f d}=\frac{V_{R}}{T_{E}}-\frac{\left(S_{E}+K_{E}\right) E_{f d}}{T_{E}},
\end{gathered}
$$

Similarly,

$$
V_{R}=\left(V_{r e f}-V_{t}-V_{3}\right)\left(\frac{K_{A}}{1+s T_{A}}\right) .
$$

Solving the above equation gives

$$
\begin{aligned}
& V_{R}=\left(V_{\text {ref }}-V_{t}\right) \frac{K_{A}}{T_{A}}-\frac{V_{3} K_{A}}{T_{A}}-\frac{V_{R}}{T_{A}} \\
& \text { and } V_{3}=V_{R}\left(\frac{s K_{F}}{1+s T_{F}}\right),
\end{aligned}
$$

Substituting the value of $R$ in above equation, we get

$$
\begin{aligned}
\forall_{3}= & \frac{K_{F} K_{A}}{T_{F} T_{A}}\left(V_{r e f}-V_{t}\right)-\left(\frac{K_{F} K_{A}}{T_{F} T_{A}}+\frac{1}{T_{F}}\right) \\
& -\left(\frac{K_{F}}{T_{F} T_{A}} V_{R}\right)
\end{aligned}
$$

The 1.0 MVA unit is modeled to regulate the speed during its normal operation, and this can be done by changing the pitch angle as a function of speed. The pitch blade control model is defined by a second-order differential equation [23].

$$
\tau_{p}^{2} \frac{d 2 \theta(t)}{d t 2}+2 \xi \tau p \frac{d \theta(t)}{d t}+\theta(t)=K_{\omega}^{\prime} \omega+K_{v}^{\prime} V+K_{\delta}^{\prime} \delta
$$

Taking Laplace transform

$$
\tau_{p}^{2} s^{2} \theta(s)+2 \xi \tau p \theta(s)+\theta(s)=K_{\omega}^{\prime} \omega+K_{v}^{\prime} V+K_{\delta}^{\prime} \delta
$$

Let $\theta_{1}=\theta(s)$

$\theta_{2}=\theta_{1}=s \theta$ 


$$
\begin{aligned}
& \tau_{p}^{2} \theta_{2} 2 \xi \tau p \theta_{2}+\theta_{1}=K_{\omega}^{\prime} \omega+K_{v}^{\prime} V+K_{\delta}^{\prime} \delta \\
& \theta_{2}=\frac{K_{\omega}^{\prime} \omega}{\tau_{p}^{2}}+\frac{K_{v}^{\prime} V}{\tau_{p}^{2}}+\frac{K_{\delta}^{\prime} \delta}{\tau_{p}^{2}}-\frac{2 \xi \tau_{p} \theta_{2}}{\tau_{p}^{2}}-\frac{\theta_{1}}{\tau_{p}^{2}}
\end{aligned}
$$

where the wind speed $V$ is the source of disturbance and contributes the variation in the generated power, it is included in the disturbance matrix $T$. The remaining terms are included in system matrix $A$. Now the seventh state is therefore defined as

$\theta_{1}=\theta_{2}$,

and the eighth state is defined as

$$
\theta_{2}=\frac{\mathrm{K}_{\omega}^{\prime} \omega}{\tau_{\mathrm{p}}^{2}}+\frac{\mathrm{K}_{\delta}^{\prime} \delta}{\tau_{\mathrm{p}}^{2}}-\frac{\theta_{1}}{\tau_{\mathrm{p}}^{2}}-\frac{2 \xi \theta_{2}}{\tau_{\mathrm{p}}} .
$$

The two states $\theta_{1}$ and $\theta_{2}$ are restructured for the blade pitch angle $\theta$.

\subsection{Optimal Regulators with Full-state Vector Feedback Control Strategy}

The vector and system matrices obtained for the design of optimal regulator are as follows:

$$
\begin{aligned}
& A=\left[\begin{array}{lccccccc}
0 & 377 & 0 & 0 & 0 & 0 & 0 & 0 \\
\left(\frac{K_{1}}{M}\right) & \left(\frac{K_{\omega}-D}{M}\right) & \left(\frac{-K_{2}}{M}\right) & 0 & 0 & 0 & \frac{K_{\theta}}{M} & 0 \\
\left(\frac{-K_{A}}{T_{d o}}\right) & 0 & \left(\frac{-1}{K_{s} T_{d 0}}\right) & \frac{1}{T_{d o}} & 0 & 0 & 0 & 0 \\
0 & 0 & 0 & \left(-\left(S_{E}+K_{E}\right) / T_{E}\right) & \frac{1}{T_{1}} & 0 & 0 & 0 \\
\left(\frac{-K_{A} K_{5}}{T_{A}}\right) & 0 & \left(\frac{-K_{A} K_{6}}{T_{A}}\right) & 0 & \frac{1}{T_{A}} & \frac{-K_{A}}{T_{A}} & 0 & 0 \\
\left(\frac{-K_{A} K_{S} K_{F}}{T_{A} T_{F}}\right) & 0 & \left(\frac{-K_{A} K_{S} K_{F}}{T_{A} T_{F}}\right) & 0 & \left(\frac{-K_{F}}{T_{A} T_{F}}\right) & \left(\frac{-\left(T_{A}+K_{A} K_{F}\right)}{T_{A} T_{F}}\right) & 0 & 0 \\
0 & 0 & 0 & 0 & 0 & 0 & 0 & 1 \\
\frac{K_{E}}{\tau_{p}^{2}} & \frac{K_{e s}}{\tau_{p}^{2}} & 0 & 0 & 0 & 0 & \frac{-1}{\tau_{p}^{2}} & \frac{-2 s}{\tau_{p}}
\end{array}\right], \\
& B^{T}=\left[\begin{array}{llllllll}
0 & 0 & 0 & 0 & \frac{K_{A}}{T_{A}} & \frac{K_{A} K_{F}}{T_{A} T_{F}} & 0 & 0
\end{array}\right], \\
& T^{T}=\left[\begin{array}{llllllll}
0 & \frac{K_{v}}{M} & 0 & 0 & 0 & 0 & 0 & \frac{K_{v}}{\tau_{p}^{2}}
\end{array}\right] \text {, } \\
& X^{T}=\left[\begin{array}{llllllll}
\delta & \omega & E_{q} & E_{f d} & V_{R} & V_{3} & \theta_{1} & \theta_{2}
\end{array}\right], \\
& D=V_{\text {gust }}, u=V_{\text {ref } f} \text {. }
\end{aligned}
$$

\subsection{Optimal Regulators with Output Vector Feedback Control Strategy}

The structures of state, control, and disturbance vectors will remain same. However, the structure of output matrix $C$ is constructed based on the number of states chosen for feedback. In the study, three sets of state variables are considered to design the optimal regulators using output vector feedback control strategy. The case studies are identified next. 
1. Case Study 1: Two states, i.e., $\delta$ and $\omega$, are considered for feedback:

$$
\left[Y_{1}\right]=\left[\begin{array}{ll}
\delta & \omega
\end{array}\right]^{T}
$$

2. Case Study 2: Four states (i.e., $\delta, \omega, E_{q}$, and $E_{f d}$ ) are considered for feedback:

$$
\left[Y_{2}\right]=\left[\begin{array}{llll}
\delta & \omega & E_{q}^{\prime} & E_{f d}
\end{array}\right]^{T} .
$$

3. Case Study 3: Five states (i.e., $\delta, \omega, E_{q}, E_{f d}$, and $V_{R}$ ) are considered for feedback:

$$
\left[Y_{3}\right]=\left[\begin{array}{lllll}
\delta & \omega & E_{q}^{\prime} & E_{f d} & V_{R}
\end{array}\right]^{T} .
$$

Similarly, the structures of $C$ matrix for Case Studies $1-3$ are

$$
\begin{aligned}
C_{1}= & {\left[\begin{array}{llllllll}
1 & 0 & 0 & 0 & 0 & 0 & 0 & 0 \\
0 & 1 & 0 & 0 & 0 & 0 & 0 & 0
\end{array}\right], } \\
C_{2}= & {\left[\begin{array}{llllllll}
1 & 0 & 0 & 0 & 0 & 0 & 0 & 0 \\
0 & 1 & 0 & 0 & 0 & 0 & 0 & 0 \\
0 & 0 & 1 & 0 & 0 & 0 & 0 & 0 \\
0 & 0 & 0 & 1 & 0 & 0 & 0 & 0
\end{array}\right], } \\
C_{3}= & {\left[\begin{array}{llllllll}
1 & 0 & 0 & 0 & 0 & 0 & 0 & 0 \\
0 & 1 & 0 & 0 & 0 & 0 & 0 & 0 \\
0 & 0 & 1 & 0 & 0 & 0 & 0 & 0 \\
0 & 0 & 0 & 1 & 0 & 0 & 0 & 0 \\
0 & 0 & 0 & 0 & 1 & 0 & 0 & 0
\end{array}\right] . }
\end{aligned}
$$

\subsection{Design Matrices}

State cost weighting matrix $Q$ and control cost weighting matrix $R$ are selected as identity matrices of appropriate dimension.

\section{Simulation Results}

In the present work, optimal controllers are designed for a wind turbine generator supplying an infinite bus through a transmission line using an output vector feedback control technique. Table 1 shows the optimum performance index values for the designed optimal regulators, whereas Table 2 describes the optimal feedback gains. The closed-loop system eigenvalues are computed to investigate the dynamic stability of the system and are shown in Table 3. The designed optimal output feedback regulators are implemented, and the system dynamic responses for various system states are obtained and compared with that obtained with optimal regulator designed using full-state vector feedback control technique. The response plots obtained are shown in Figures 4-15. Figures 4-7 show the response plots obtained for optimal regulators using the feedback of system states as identified in Case Study 1. Figures 8-11 show the response plots obtained for optimal regulators using the feedback of four states $\left(\delta, \omega, E_{q}\right.$, and $\left.E_{f d}\right)$ for Case Study 2 . Figures $12-15$ show the 
response plots obtained for optimal regulators using the output feedback control strategy for Case Study 3.

Table 1 Optimum performance index values

\begin{tabular}{|c|c|c|c|}
\hline \multicolumn{4}{|l|}{ Optimal } \\
\hline regulators using & \multicolumn{3}{|c|}{ Optimal regulators with output vector feedback } \\
\hline \multicolumn{4}{|l|}{ full-state vector } \\
\hline feedback & Case Study 1 & Case Study 2 & Case Study 3 \\
\hline 149.3600 & 119.0185 & 94.2110 & 147.5100 \\
\hline
\end{tabular}

Table 2 Optimal feedback gain matrices

\begin{tabular}{|c|c|c|}
\hline \multicolumn{2}{|c|}{ Optimal regulators using full-state feedback } & [25.8-5217.5 $47.11 .0-0.929 .4-154.254 .4]$ \\
\hline Optimal regulators & Case Study 1 & {$\left[\begin{array}{llllllllll}-0.6803 & -77.8905 & 0.3446 & 0.0042 & 0.0000 & -0.0010 & 5.3264 & 1.1534\end{array}\right]$} \\
\hline with output & Case Study 2 & [29.8-3737.4 38.0 $1.00 .0-0.0-167.537 .4]$ \\
\hline vector feedback & Case Study 3 & {$\left[\begin{array}{lllllll}25.6 & -5174.9 & 46.9 & 1.0 & -0.9 & 29.5-152.8 & 53.8]\end{array}\right.$} \\
\hline
\end{tabular}

\section{Discussion of Results}

The examination of optimum performance index values reveals that optimum performance index value varies as the system states and the number of system states selected for the feedback changes. A reduction in performance index value is exhibited while using an output vector feedback control strategy as compared to that obtained with a full-state vector feedback control strategy. The performance index value is a prime index to reflect the cost effectiveness of the designed controllers.

Table 3 Closed-loop system eigenvalues

\begin{tabular}{|c|c|c|c|}
\hline Optimal gains & \multicolumn{3}{|c|}{ Optimal gains with output vector feedback } \\
\hline \multicolumn{4}{|l|}{ with full-state } \\
\hline feedback & Case Study 1 & Case Study 2 & Case Study 3 \\
\hline$-20,009$ & -651 & $-1+6 i$ & $-1-6 i$ \\
\hline$-5+5 i$ & $-5-5 i$ & -5 & $\overline{-1}$ \\
\hline-650.93 & $-10.02+24.85 i$ & $-10.02-24.85 i$ & $-24.15+10.28 i$ \\
\hline$-24.15-10.28 i$ & $-4.71+4.72 i$ & $-4.71-4.72 i$ & -0.10 \\
\hline$-20,000$ & -651 & $-1+6 i$ & $-1-6 i$ \\
\hline$-5+5 i$ & $-5-5 i$ & -1 & -0 \\
\hline$-20,009$ & -651 & $-1+6 i$ & $-1-6 i$ \\
\hline$-5+5 i$ & $-5-5 i$ & -5 & -1 \\
\hline
\end{tabular}




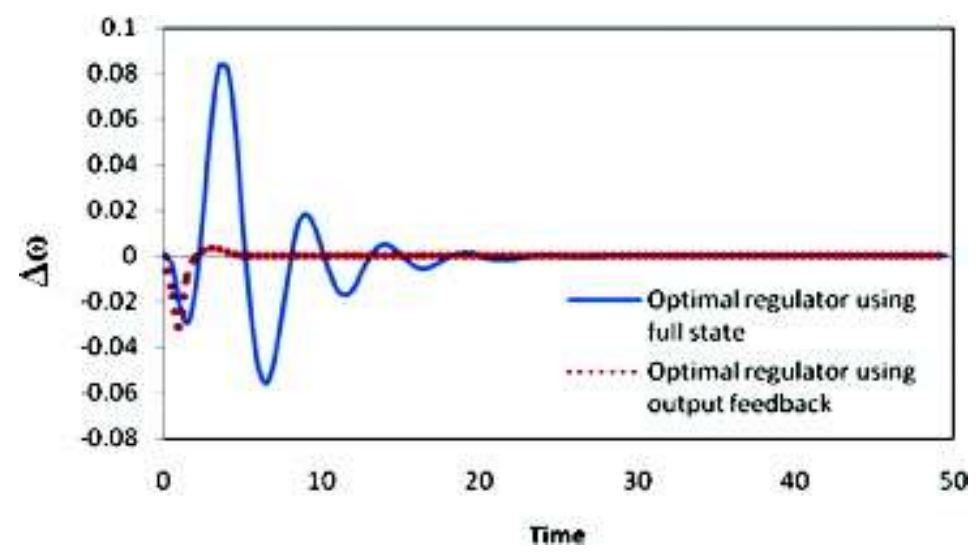

FIGURE 4. Dynamic response of $\Delta \omega$ for Case Study 1.

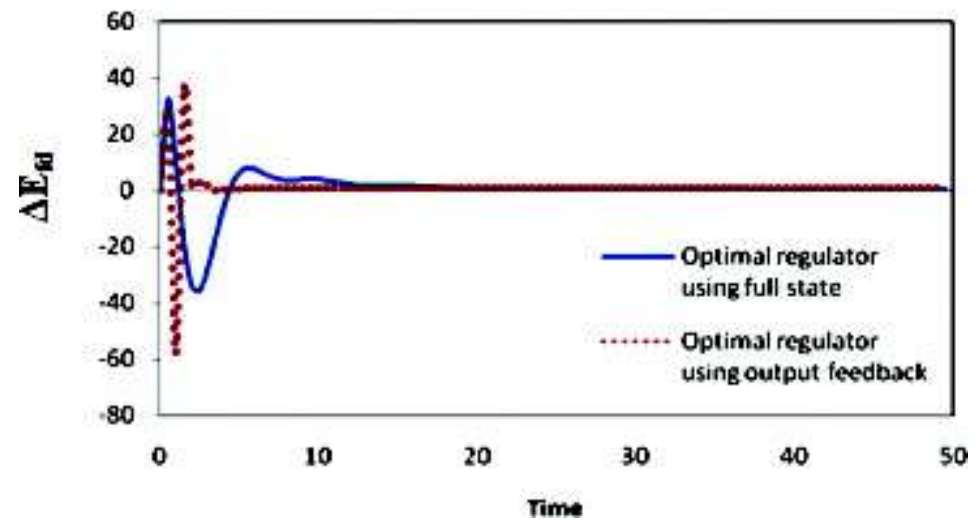

FIGURE 5. Dynamic response of $\Delta E_{f d}$ for Case Study 1.

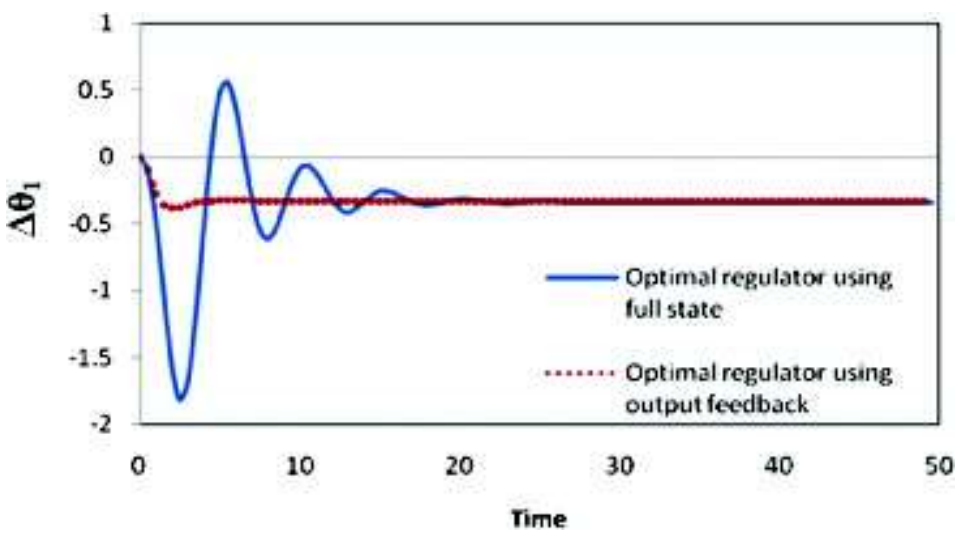

FIGURE 6. Dynamic response of $\Delta \theta_{1}$ for Case Study 1 . 


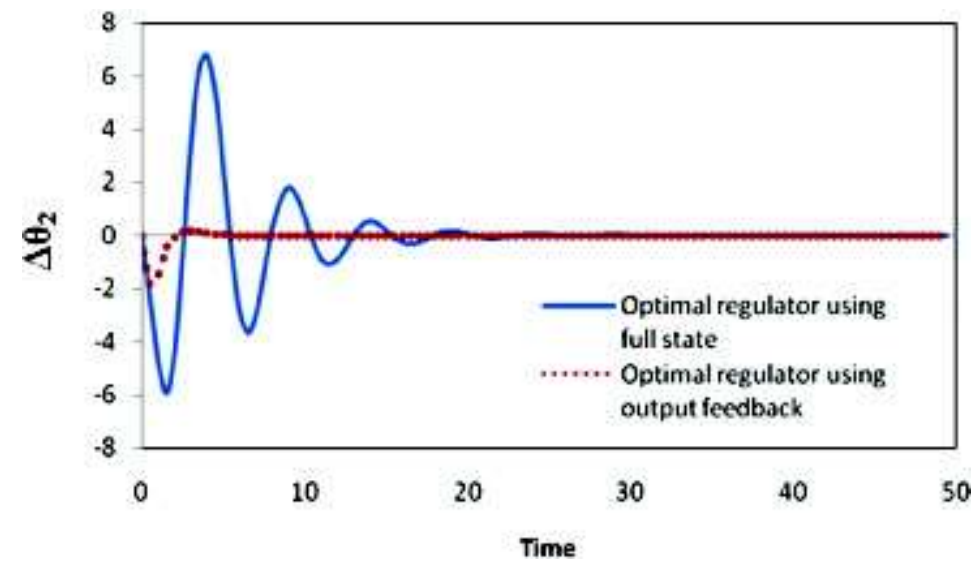

FIGURE 7. Dynamic response of $\Delta \theta_{2}$ for Case Study 1.

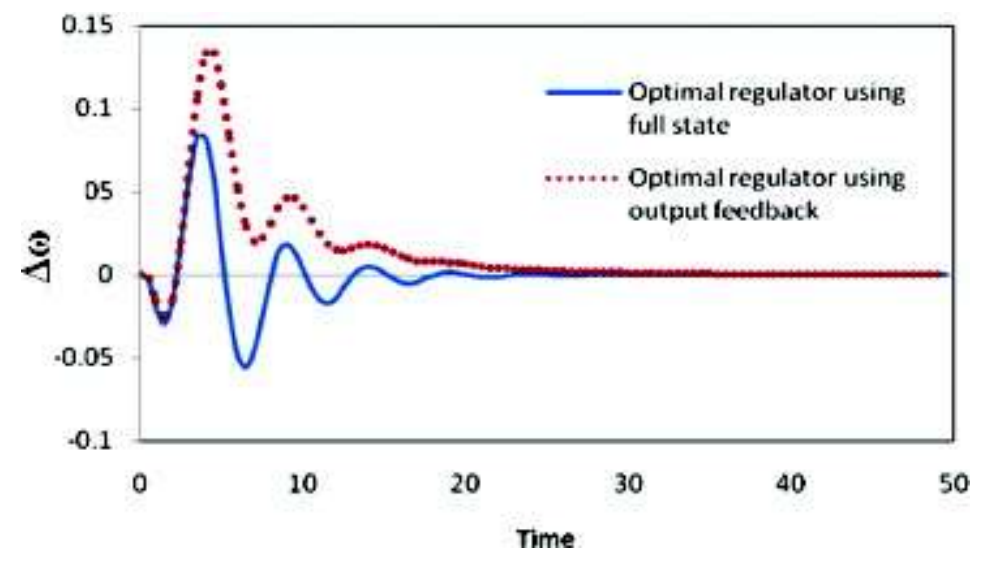

FIGURE 8. Dynamic response of $\Delta \omega$ for Case Study 2.

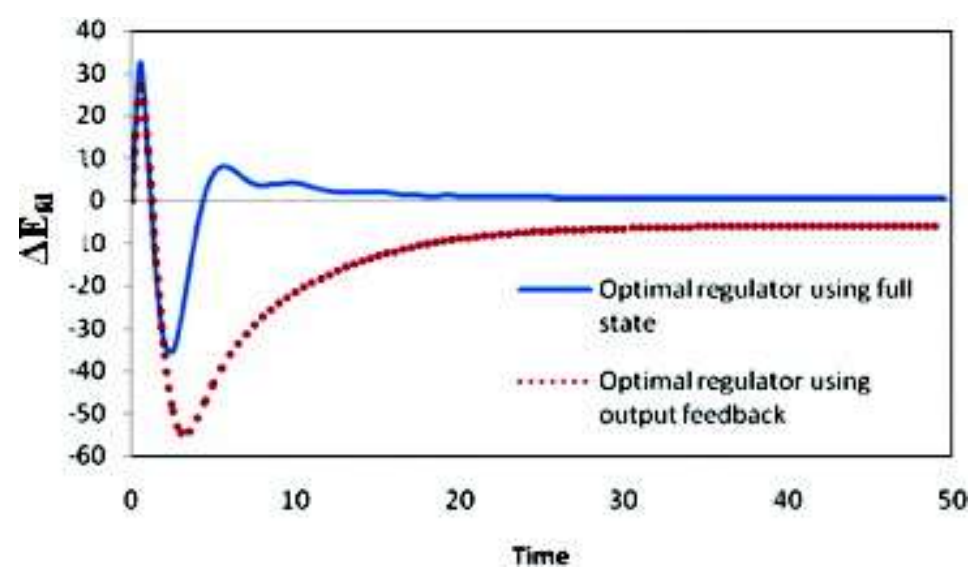

FIGURE 9. Dynamic response of $\Delta E_{f d}$ for Case Study 2. 


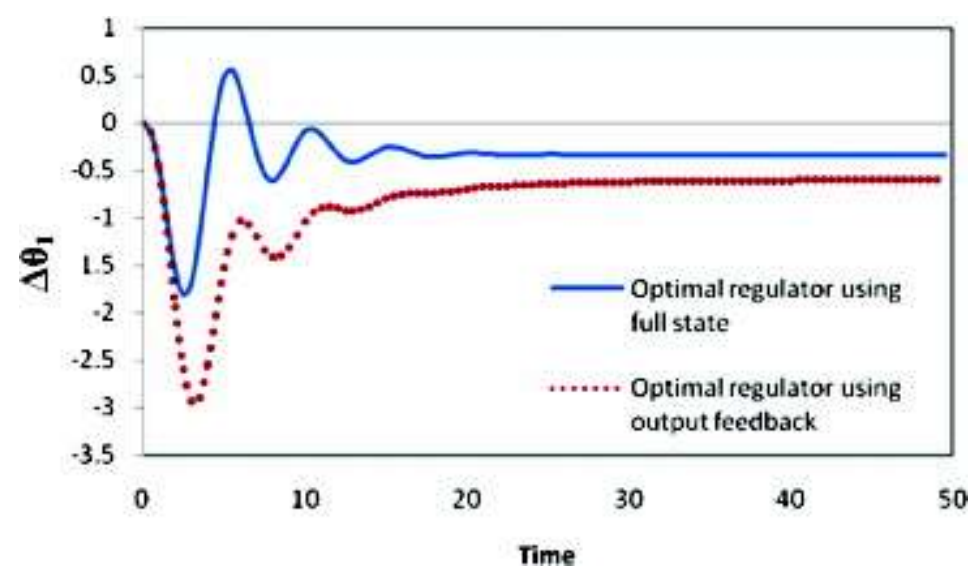

FIGURE 10. Dynamic response of $\Delta \theta_{1}$ for Case Study 2.

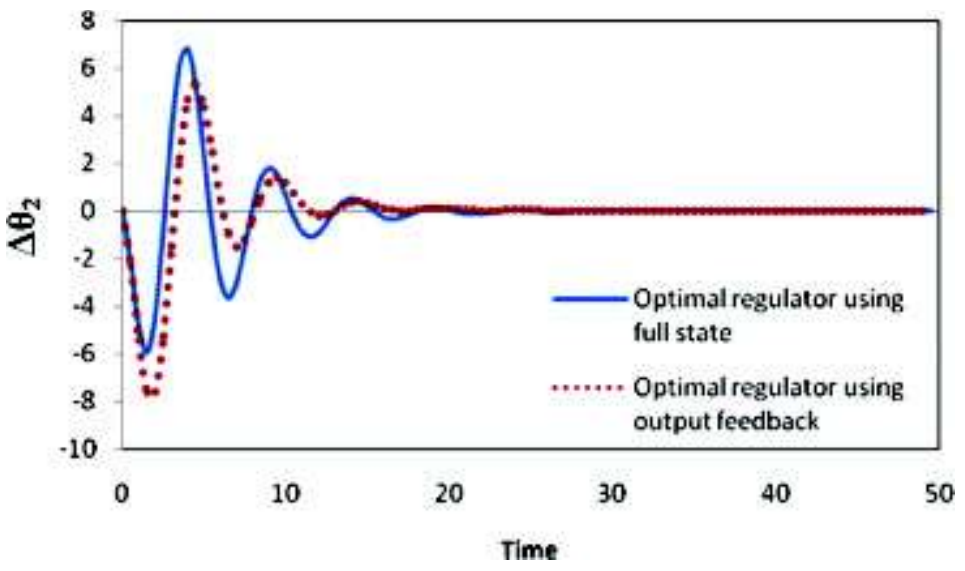

FIGURE 11. Dynamic response of $\Delta \theta_{2}$ for Case Study 2.

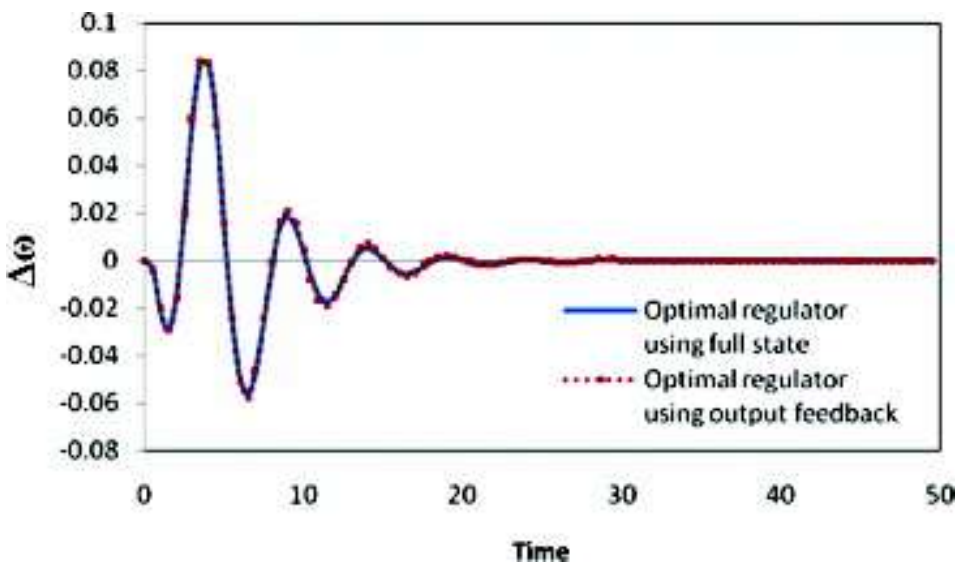

FIGURE 12. Dynamic response of $\Delta \omega$ for Case Study 3. 


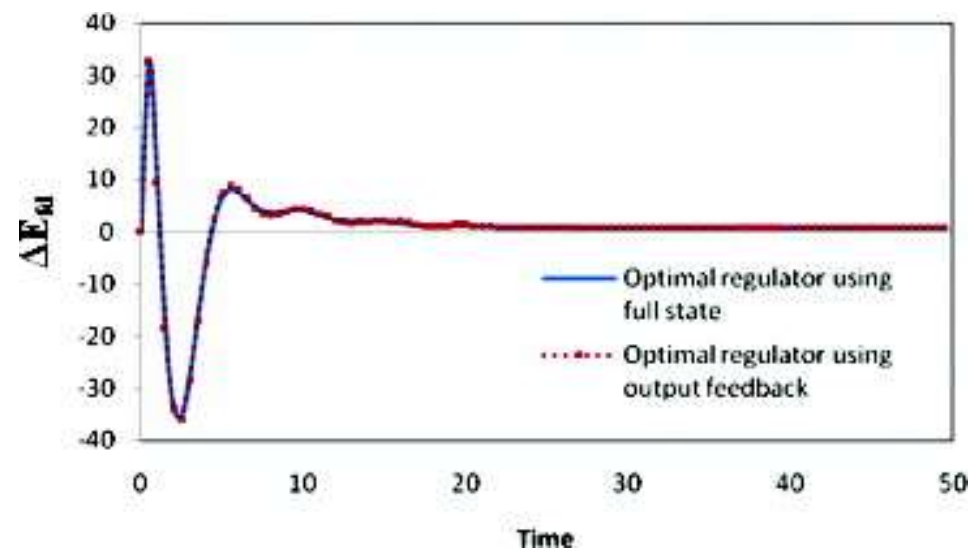

FIGURE 13. Dynamic response of $\Delta E_{f d}$ for Case Study 3.

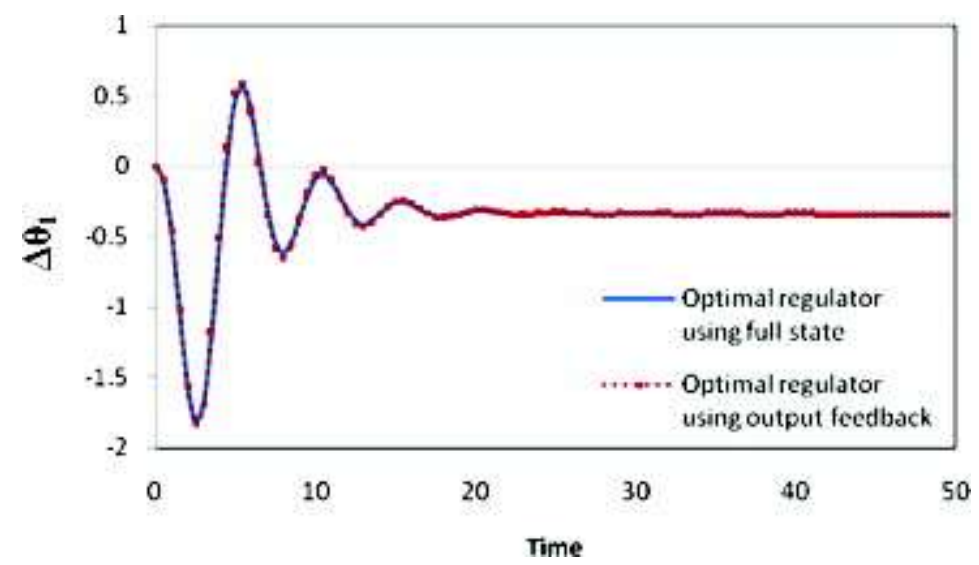

FIGURE 14. Dynamic response of $\Delta \theta_{1}$ for Case Study 3.

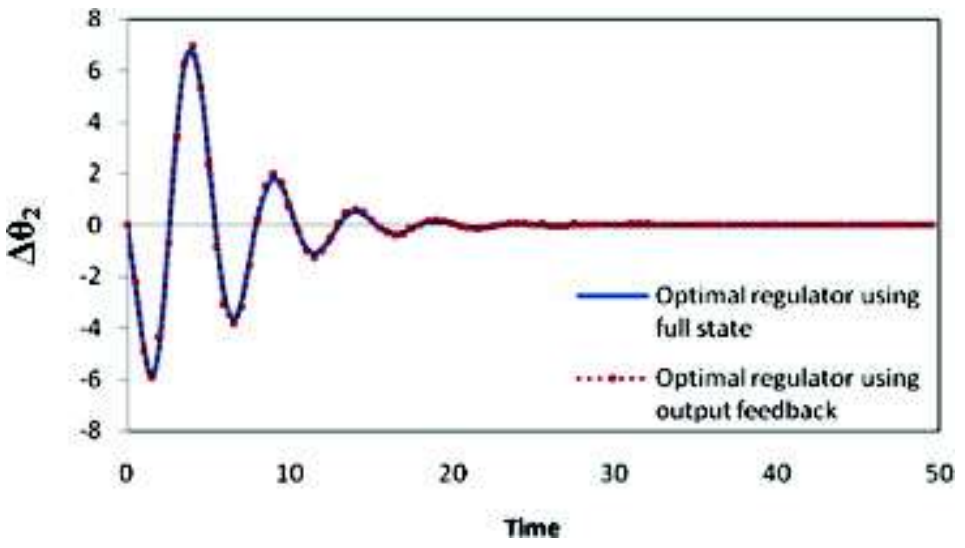

FIGURE 15. Dynamic response of $\Delta \theta_{2}$ for Case Study 3.

From the assessment of patterns of closed-loop system eigenvalues, it can be divulged that an increase in values of real and imaginary parts obtained in Case Study 1 using output vector feedback control led to the improvement in system dynamic stability margins available for the system. The 
reduction in the real part of some of the eigenvalues is obtained in Case Study 2 using the output vector feedback as compared to full-state vector feedback, leading to deterioration in the dynamic performance of the system. However, similar patterns of closed-loop system eigenvalues are obtained in Case Study 3 as that obtained with full-state vector feedback. Therefore, it can be revealed that the states identified in Case Study 3 as output variables are dominating or are important as compared to other states. Also, using these outputs as feedback for controller designs, almost similar system dynamic performance is provided by controllers designed using the feedback of all system states (full state). Moreover, the closed-loop system is stable for all case studies carried out considering various sets of state variables for feedback.

As shown in the response plots of Figures $4-7$, while only the first two states, i.e., $\delta$ and $\omega$, are considered as output states for feedback, there is an appreciable reduction in the magnitudes of the first peak of responses as compared to those obtained with optimal regulators designed based on the full-state feedback technique. However, system response for $E_{f d}$ is sluggish and associated with large first overshoot with regulators designed in Case Study 1 . The investigations of the time response plots of Figures 8-11 reveal that there is a high increase in the magnitudes of the first peak of responses achieved with the implementation of optimal regulators designed using the output feedback control technique, while the first four states $\left(\delta, \omega, E_{q}\right.$, and $E_{f d}$; i.e., Case Study 2$)$ are considered for feedback. Interestingly, again, almost comparable system dynamic performance is obtained, as shown in Figures 12-15, with the implementation of designed optimal regulators using feedback of the first five states $\left(\delta, \omega, E_{q}, E_{f d}\right.$, and $\left.V_{R}\right)$ for controller design (i.e., Case Study 3 ) as compared to that obtained with optimal controllers designed using full-state vector feedback.

\section{Conclusion}

This article investigates the design and implementation feasibility of optimal controllers based on the output vector feedback control technique. The controllers are designed for a wind generator supplying an infinite bus through a transmission line. The system dynamic performance has been investigated with the implementation of designed controllers when the system is perturbed. From the investigations carried out, the following can be concluded.

- The optimal controller designs using the output vector feedback control technique result in a reduction in optimum performance index values, demonstrating the cost effectiveness of the designs as compared to that of the full-state vector feedback control technique.

- The closed-loop system stability is ensured for all case studies carried out to design optimal regulators using the output vector feedback control strategy.

- Further, output feedback controllers can perform satisfactorily in real-world power systems where access to system states is limited. The set of five states $\left(\delta, \omega, E_{q}, E_{f d}\right.$, and $\left.V_{R}\right)$ is identified as the best feasible set of state variables that can be used for feedback to get almost the same system dynamic performance as achieved with optimal regulators designed with the full-state vector feedback control strategy.

\section{Appendix: Numerical Data}

$\begin{array}{llll}x_{d}=2.21, & \mathrm{x}^{\prime}{ }_{d}=0.165, & x_{q}=1.064, & M=19.04, \\ D=0, & \omega_{o}=377, & T_{d o}=1.942, & x_{e}=0.3, \\ K_{A}=400, & T_{A}=0.02, & K_{E}=1, & T_{E}=1.3, \\ K_{F}=0.03, & T_{F}=1.0, & S_{E}=0.64, & K_{v}^{\prime}=-0.337, \\ K_{\omega}^{\prime}=-20.94, & K_{\delta}^{\prime}=-0.0055, & \tau_{p}=0.15, & \xi=0.707, \\ K_{\omega}=-3.3, & K_{\theta}=0.118, & K_{v}=0.337, & V=7.92, \\ T=4, & G_{u}=0.075 . & & \end{array}$




\section{NOMENCLATURE}

A $\quad n \times \mathrm{n}$ system matrix

$B \quad n \times m$ input matrix

D damping coefficient

$E_{f d} \quad$ generator field voltage

$E_{q}^{\prime} \quad$ voltage proportional to direct axis flux linkage

$I_{d o}, I_{q o} \quad \mathrm{~d}$ - and q-axis components of armature current

$J$ performance index

$K_{A} \quad$ regulator gain

$K_{F} \quad$ gain of excitation system stabilizer

$K_{\omega}, K_{v}, K_{\delta}$ pitch angle regulation constants

M moment of inertia

$P_{i} \quad$ mechanical input power

$R_{e} \quad$ system shunt resistance

$S_{E} \quad$ saturation function

$T \quad n \times 1$ disturbance matrix

$T_{A} \quad$ regulator time constant

Te electric torque

$T_{F} \quad$ time constant of excitation system stabilizer

V wind speed

$V_{d o}, V_{q o} \quad d$ - and $q$-axis components of terminal voltage

$V_{R} \quad$ regulator voltage

$V_{t} \quad$ terminal voltage

$X_{d}, X_{q} \quad d$ - and $q$-axis components of synchronous reactance

$X_{d}^{\prime} \quad$ transient reactance of machine in $d$-axis

$\delta \quad$ angle between $q$-axis of the generator and infinite bus

$\theta \quad$ blade pitch angle 

$\theta_{1}, \theta_{2} \quad$ reconstructed states for blade pitch angle
$\xi \quad$ damping ratio
$\mathrm{T}_{p} \quad$ actuator time constant
$\omega_{0} \quad$ angular rotor speed at operating point

\section{References}

1. Hinrichsen, E.N., and Nolan, P.J., "Dynamics and stability of wind turbine generators," IEEE Trans. Power Apparat. Syst., Vol. 101, No. 8, pp. 2640-2648, 1982.

2. Johnson, C.C., and Smith, R.T., "Dynamics of wind generators on electric utility networks," IEEE Trans. Aerospace Electron. Syst., Vol. 12, No. 4, pp. 483-492, 1976.

3. Jorgensen, G.E., Lotker, M., Meier, R.C., and Brierley, D., "Design, economic and system considerations of large wind driven generators," IEEE Trans. Power Apparat. Syst., Vol. 95, pp. 870878, 1976.

4. Wasynczuk, O., Man, D.T., and Sullivan, J.P., "Dynamic behavior of a class of wind turbine generators during random wind fluctuation," IEEE Trans. Power Apparat. Syst., Vol. 100, No. 6, pp. 2837-2845, 1981.

5. Rau, V.G., and Durga Prasad, G., "Dynamic stability assessment of wind turbine generators using the Lyapunov function approach," Electr. Power Syst. Res., Vol. 27, No. 1, pp. 61-72, 1993.

6. Abdel-Magid, Y.L., and El-Amin, I.M., "Dynamic stability of wind-turbine generators under widely varying loading conditions," Int. J. Electr. Power Energy Syst., Vol. 9, No. 3, pp. 180-188, 1987.

7. Wang, J.C., and McCalley, D., "Impactofwindpoweroncontrolperformancestandards," Int. J. Electr. Power Energy Syst., Vol. 47, pp. 225-234, May 2013.

8. Hui, L., Shengquan, L., Haiting, J., Yang, D., Yang, C., Chen, H., Zhao, B., Hu, Y., and Chen, Z., "Dampingcontrol-strategiesofinter-arealow-frequencyoscillationforDFIG-basedwindfarmsintegratedintoapowersystem," Int. J. Electr. Power Energy Syst., Vol. 61, pp. 279-287, October 2014.

9. Ise, T., Miura, Y., and Bevrani, H., "Virtual synchronous generators: A survey and new perspectives," Int. J. Electr. Power Energy Syst., Vol. 54, pp. 244-254, 2014.

10. Anderson, B.D. O., and Moore, J.B., "The standard regulator problem-II," in Optimal Control: Linear Quadratic Methods, Englewood Cliffs, NJ: Prentice Hall, pp. 35-51, 1989.

11. Ibraheem and Kumar, P., "A novel approach to matrix Riccati equation solution: An application to optimal control of interconnected power systems," Electr. Power Compon. Syst., Vol. 32, No. 152, January 2004.

12. Yahagi, T., "Optimal output feedback with reduced performance index sensitivity," Int. J. Control, Vol. 25, No. 5, pp. 769-783, 1977.

13. Fekiha, A., and Chowdhury, F.N., "A nonlinear state feedback controller for induction motors," Electr. Power Compon. Syst., Vol. 33, No. 11, pp. 1211-1227, 2006. 
14. Sundareswarana, K., and Sreedevi, V.T., "Design and development of feed-back controller for a boost converter using a colony of foraging bees," Electr. Power Compon. Syst., Vol. 37, No. 5, pp. 465-477, 2009.

15. Ani, V.A., "Optimal control of PV/wind/ hydro-diesel hybrid power generation system for off-grid macro base transmitter station site," Electron. J. Energy Environ., Vol. 1, No. 2, pp. 37-55, August 2013.

16. Javier, S.G., Burgos Payan, M., and Riquelme Santos, J., "Optimal control of wind turbines for minimizing overall wake effect losses in offshore wind farms," Proceedings of IEEE European Conference 2013 (EUROCON 2013), Zagreb, Croatia, 1-4 July 2013.

17. Yan, Z., Hall, J., and Dongmei, C., "MIMO control of wind turbine using direct shooting method," Proceedings of the American Control Conference (ACC), Washington DC, 17-19 June 2013.

18. Hong, C.M., Cheng, F.S., and Chen, C.H., "Optimal control for variable-speed wind generation systems using general regression neural network," Int. J. Electr. Power Energy Syst., Vol. 60, pp. 1423, 2014 .

19. Battista, H.D., Mantz, R.J., and Christiansen, C.F., "Energy-based approach to the output feedback control of wind energy systems," Int. J. Control, Vol. 76, No. 3, pp. 299-308, 2003.

20. Thomas, R., Puthoff, R., Savino, J., and Johnson, W., "Plan and status of the NASA-Lewis Research Center wind energy projects," Proceedings of the Joint IEEE/ASME Power Conference, Paper No. NTIS N75-21795, Portland, OR, 1975.

21. Al-Duwaish, H.N., Al-Hamouz, Z.M., and Badran, S.M., "Adaptive output feedback controller for wind turbine generators using neural networks," Electr. Machines Power Syst., Vol. 27, pp. 465-479, 1999.

22. Bellman, R., "Matrices and differential equations," in Introduction to Matrix Analysis, New York: McGraw-Hill, Chap. 10, pp. 159-182, 1960.

23. Hwang, H., and Gilber, L.J., "Synchronization of wind turbine generators against an infinite bus under gusting conditions," IEEE Trans. Power Apparat. Syst., Vol. 97, pp. 536-544, 1978.

\section{Author biographies}

Shahida Khatoon obtained her B.Tech (electrical) in 1990 from Jamia Millia Islamia (JMI), New Delhi, India, and her M.Tech. (control and instrumentation) from I.I.T. Delhi in 1995. She was awarded her Ph.D. with specialization in autonomous robotics and control from JMI. She has been working as a professor in the Department of Electrical Engineering, JMI, for the last 20 years. She has credited in her name many research papers in refereed journals and conferences. She is actively involved in guiding Ph.D. students and installing research projects sponsored by University Grants Commission and Department of Science and Technology. She was conferred the prestigious All India Council of Technical Education (AICTE) Award for Young Teachers in 2008. She has also worked at AT \& T Bell Lab, New Jersey, USA in 2004. Her research interests include electric power systems and wind energy.

Ibraheem Nasiruddin received his B.Sc.Engg. (hons.), M.Sc.Engg., and Ph.D. in electrical engineering from Aligarh Muslim University, Aligarh, India, in 1982, 1987, and 2000, respectively. He has been working with the Department of Electrical Engineering, Jamia Millia Islamia (Central University), since January 1988, discharging his duties as the head of the Department of Electrical Engineering during 2002 to 2005. Presently he is working with the Department of Electrical Engineering, Al-Qassim University, Al-Qassim, Saudi Arabia. He is a member of various academic societies of national and international repute. He has published over 150 research papers in national/international journals/conferences. He is the recipient of Gold Medal from the Union Ministry of Power and Energy, India, in 1998 for one of his published research articles. His current activities 
include teaching and research in the areas of power system control, optimal control theory, optimal and suboptimal control of power systems, applications of model order reduction techniques, and soft computing techniques in power systems

Reshma Ehtesham received her B.Tech. (electrical engineering) and M.Tech. (electrical power system management) from Jamia Millia Islamia (JMI), New Delhi, India, in 1995 and 2009, respectively. She is currently pursuing her Ph.D. in the Department of Electrical Engineering, JMI, New Delhi, India. She has worked as an assistant professor in Priyadarshini College of Computer Sciences, Uttar Pradesh Technical University, U.P., India, from 1999 to 2006. She has published a few research articles in national and international conferences. Her research interests include electric power systems and wind energy.

Ramesh C. Bansal has more than 25 years of teaching, research, and industrial experience. Currently he is the professor and group head (power) in the Department of Electrical, Electronic and Computer Engineering at University of Pretoria, South Africa. He was previously with University of Queensland, Australia; Birla Institute of Technology and Science, Pilani, India; University of the South Pacific, Fiji; and Civil Construction Wing, All India Radio. He has published over 200 papers in journals and conferences. He is an editor/associate editor of member many reputed journals, including IET-Renewable Power Generation, IEEE Access, and Electric Power Components and Systems. He is a Fellow and CEngg IET-UK, Fellow Engineers Australia, Fellow Institution of Engineers (India), and Senior Member of IEEE. He has diversified research interests in the areas of renewable energy and conventional power systems, which includes wind, photovoltaic (PV), hybrid power systems, distributed generation, grid integration of renewable energy, power systems analysis (reactive power/voltage control, stability, faults and protection), smart grid, flexible AC transmission systems (FACTS), and power quality. 